\title{
Papel de la economía en la política sanitaria, gestión sanitaria y la práctica clínica
}

\author{
Posición de la Asociación de Economía de la Salud ${ }^{a}$ \\ en relación a la necesidad de introducir la Economía \\ de la Salud en la Formación de Pregrado en Medicina.
}

\author{
Role of Economy in the Health Policy, Health Management and Clinical Practice
}

Asociación de Economía de la Salud

La adecuada comprensión del nivel de salud requiere considerar conjuntamente las características individuales y la interacción con el entorno en el que viven las poblaciones, su capacidad de desarrollo socioeconómico y su sistema de protección social. Los ejemplos de la influencia del entorno socioeconómico en la salud son numerosos, y no es preciso acudir a ejemplos extremos ${ }^{1,2}$, para encontrar evidencias empíricas de esta relación en nuestro entorno ${ }^{3,4}$.

La Economía se ha convertido en un área de conocimiento claramente influyente en las Políticas de Bienestar (entre ellas las de protección social), y en la Gestión ${ }^{5,6,7}$, adquiriendo valor creciente en aspectos cruciales, como los relacionados con las decisiones clínicas y las preferencias individuales.

En el caso particular de los Sistemas de Salud, la Economía -Economía de la Salud- ha hecho aportaciones muy relevantes en los últimos 50 años. En el ámbito de las políticas se pueden destacar: 1) el aná- lisis de las características del mercado asegurador $\left.{ }^{8}, 2\right)$ la aproximación teórica sobre el establecimiento de prioridades en el acceso a los servicios ${ }^{9}, 3$ ) las evidencias empíricas sobre la influencia en el consumo de distintas estrategias de cobertura aseguradora ${ }^{10,11}, 4$ ) la introducción de los años de vida ajustados por calidad (AVAC) en la valoración de la eficiencia de las políticas públicas sobre la salud ${ }^{12,13}, 5$ ) las recientes aportaciones del análisis coste-beneficio generaliza$\mathrm{do}^{14}$ y 7) la constatación de que más gasto en salud no siempre produce mejores resultados ${ }^{15}$.

En el terreno de la gestión, se puede constatar la preocupación de la disciplina por mejorar el desempeño de los sistemas de salud como determinante de la misma ${ }^{16}$, y las aportaciones más precisas en el terreno de la gestión de las organizaciones sanitarias; en particular, en: 1) la determinación de los incentivos apropiados para alcanzar el justo equilibrio entre eficiencia y calidad ${ }^{17}, o$ 2) el ajuste de ries-

a. Constituida formalmente en 1985, aunque con actividad pública desde 1980, AES es una sociedad científica relacionada con la Economía, no una asociación de economistas -de hecho, más del 40\% de sus socios son médicos- www.aes.es

b. Entre los títulos a destacar, "La formación de los profesionales de la salud. Escenarios y factores determinantes" con aportaciones de Andreu Mas Colell, Beatriz González, José Conde, Guillem López y Juan Ma Cabasés, sobre los factores determinantes de la formación de los profesionales de la salud que tienen que ver con la Economía de la Salud FFBV, 1997 y "La formación de los profesionales de la salud" Formación pregraduada, postgraduada y formación continuada, informe de José M Ma Segovia de Arana, Cristóbal Pera Blanco Morales, Juan José Goiriena de Gandarias y Juan Mª Cabasés (FBBV, Enero 1999) 
gos para la financiación capitativa de los proveedores sanitarios ${ }^{18}$ o la literatura sobre coordinación, organización e incentivos asociados ${ }^{19}$.

Finalmente, en el ámbito clínico, la aportación más importante es la introducción del concepto de preferencias (utilidades) de los pacientes, sea en la evaluación económica ${ }^{20}$ sea en su incorporación a la decisión clínica ${ }^{21}$.

Desde 1985, la Asociación de Economía de la Salud (AES) resume, de algún modo, el bagaje acumulado durante años en la promoción de la investigación y la difusión de la Economía de la Salud en España.

\section{¿QUÉ DEBE SABER DE ECONOMÍA UN ESTUDIANTE DE MEDICINA?}

Según todo lo señalado, consideramos que un estudiante de medicina debe saber que la salud y la enfermedad se producen en un entorno socioeconómico donde los cuidados se prestan por instituciones con incentivos definidos y en un contexto de recursos limitados.

Ello implica tener conciencia de que la práctica clínica para resultar efectiva, deberá tratar a tiempo a los pacientes apropiados con las tecnologías más efectivas, pero además deberá tomar las decisiones más eficientes y según las preferencias de los pacientes y la sociedad.

En suma, será necesario que el estudiante de medicina incorpore a su bagaje ${ }^{22}$ :

1) el conocimiento del sistema de salud en el que va a desarrollar su actividad profesional;

2) el conocimiento de los incentivos que actúan en las organizaciones en las que va a trabajar y cómo éstos pueden influir en la producción de sus cuidados;

3) la consideración de que la ética de las decisiones clínicas exige considerar el coste de oportunidad de cada una de ellas;

4) las habilidades para la elección de las alternativas que optimicen calidad y eficiencia; que las preferencias de los pacientes deben tenerse en cuenta en la toma de decisiones clínicas.

\section{ESTADO DE LA ENSEÑANZA DE LOS PRINCIPIOS-CONOCIMIENTOS- ACTITUDES HABILIDADES DE LA ECONOMÍA EN LA FORMACIÓN PREGRADO DE MEDICINA}

Un reciente estudio promovido por The National Institutes of Health (NIH), The Robert Wood
Johnson Foundation (RWJF) y The Institute of Medicine (IOM) ha puesto de relieve la inadecuada formación de los estudiantes de medicina estadounidenses en contenidos y procedimientos relacionados con las ciencias sociales y del comportamiento. En lo referente a la Política y Economía de la Salud, concluye la necesidad de proveer un currículum que permita al alumno comprender el contexto en el que va a realizar su práctica. En particular: tener formación en el impacto social de las desigualdades en los cuidados de salud, conocer los determinantes económicos que influyen en la salud, conocer los incentivos económicos que afectan a las decisiones de los pacientes, conocer el significado del binomio coste-efectividad, entender cómo responden los médicos a los incentivos financieros y ser consciente de la presencia de variaciones de la práctica ${ }^{23}$.

Para el caso español no disponemos de estudios semejantes que orienten las políticas educativas pregrado, aunque, es conveniente resaltar que el debate sobre la formación de los profesionales de la salud se inició hace ya casi una década con diversos encuentros e informes promovidos por la Asociación de Economía de la Salud y la fundación -entonces- $\mathrm{BBV}^{\mathrm{b}}$.

Más recientemente, y consecuencia del documento sobre estándares internacionales para la Educación Médica de Pregrado que la World Federation for Medical Education $\left(\mathrm{WFME}^{24}\right.$ ) presentó en Copenhague, en octubre de 2001, se proclamó la llamada declaración de Granada sobre estándares en la Educación Médica de Pregrado ${ }^{25}$ en España. La declaración compromete a los socios a "(...) establecer un sistema de evaluación y acreditación de las facultades de medicina de ámbito nacional e internacional con el propósito de garantizar unos estándares mínimos de calidad para sus progra$\operatorname{mas}(. .)$.

Entre los compromisos que idealmente se deberían asumir, la declaración insta a las Facultades de Medicina a: "Enseñar los principios de la medicina científica y la medicina basada en la evidencia, así como el pensamiento analítico y crítico durante todo el currículum. Así mismo, además de las contribuciones de las ciencias biomédicas básicas, las facultades deben incorporar en su currículum, las contribuciones de las ciencias de la conducta y sociales, de ética médica y de Economía de la Salud que garanticen el comportamiento adecuado en el ejercicio de la práctica profesional y que propicien habilidades de comunicación, de toma de decisiones y de liderazgo e influencia social. La promoción de la salud y la prevención de la enfermedad deberán constituir el hilo conductor de todo el currículum". 
Contrastando con este compromiso, la Asociación de Economía de la Salud, ha constatado que:

1) La enseñanza pregrado de la Economía de la Salud queda actualmente relegada a ocupar un espacio casi anecdótico entre los contenidos de la asignatura de Medicina Preventiva y Salud Pública y en algunos cursos con créditos de libre elección ${ }^{c}$;

2) En la actual propuesta de títulos universitarios de medicina como desarrollo del Real Decreto $55 / 2005$, de 21 de enero, no aparecen contenidos específicos de la disciplina.

3) La inexistencia de formación en Economía de la Salud, Política y Gestión Sanitaria nos sitúa al margen de la tendencia ya consolidada en países desarrollados donde su incoporación ha supuesto una contribución decisiva a la mejora en la toma de decisiones.

\section{DECLARACIÓN}

Dada la relevancia de la Economía de la Salud en el desarrollo y la calidad del Sistema Nacional de Salud español y la escasa presencia de la disciplina en la formación pregrado de los nuevos profesionales, la Asociación de Economía de la Salud (AES) considera que:

1. La Economía de la Salud, es una disciplina consolidada, con importantes aportaciones al conocimiento y desarrollo del Sistema de Salud.

2. En España, la Economía de la Salud, encarnada por profesionales de distintos campos, ha contribuido decididamente a la mejora del Sistema; la Asociación de Economía de la Salud (AES) cuenta con 30 años de experiencia y ha sido el catalizador de una buena parte de las acciones.

3. En la actualidad, la formación de pregrado de los profesionales sanitarios españoles, no aborda conocimientos, actitudes y habilidades relacionadas con la Economía de la Salud, la Política Sanitaria y la Gestión que resultan imprescindibles para el adecuado desarrollo profesional posterior.

4. Que en el actual proceso de convergencia educativa (espacio de Bolonia) la incorporación explícita de contenidos conceptuales y habilidades en el currículum de la formación pregrado de profesiones sanitarias es imprescindible. Los Ministerios de Educación y Ciencia y Sanidad y Consumo, así como

c. Dos ejemplos de esta formación de libre elección se encuentran en la Universidad Autónoma de Madrid

(http://www.uam.es/centros/medicina/cursodegestionsanitaria.doc) y en la Universidad de Oviedo

(http://directo.uniovi.es/Catalogo/FichaAsignatura.ASP?asignatura=5965). las Universidades Públicas y Privadas deben velar por la incorporación de los contenidos de Economía de la Salud en la regulación de los estudios de Medicina y para ello dotar de los recursos humanos y materiales necesarios en sus Facultades

La Asociación de Economía de la Salud desea contribuir activamente a la consecución de los objetivos señalados en esta declaración y al mismo tiempo ofrece la oportunidad de que otras sociedades científicas que compartan su contenido se sumen a ella.

\section{BIBLIOGRAFÍA}

1. Banco Mundial. Informe sobre el desarrollo mundial 1993. Invertir en salud. Washington: Banco Mundial, 1993.

2. Sen, A ¿Por qué la equidad en salud?. Rev Panam Salud Publica, vol.11, no.5, p.302-309. ISSN 1020-4989

3. Inequalities in Health Report of a Research Working Group. 1980 http://www.sochealth.co.uk/history/black.htm

4. van Doorslader, E. and C. Masseria (2004), .Income-Related Inequality in the Use of Medical Care in 21 OECD Countries., OECD Health Working Paper, No. 14, 11 May 2004, OECD, Directorate for Employment, Labour and Social Affairs, Paris.

5. Ortún V, Meneu R Impacto de la Economía en la Política y Gestión Sanitaria. Rev Esp Salud Pública 2006; 80: 491-504.

6. Ortún V El impacto de la Economía de la Salud en la Política y en la Gestión. Economía y Salud, $N^{\circ}$ especial sobre 25 años de Economía de la Salud, Mayo 2005, p.19-22.

7. González López-Valcárcel B ¿Qué hace la Economía por nuestra Salud?. Economía y Salud, $\mathrm{N}^{\circ}$ especial sobre 25 años de Economía de la Salud, Mayo 2005, p. 27-32.

8. Arrow $\mathrm{K}$ "Uncertainty and the Welfare Economics of Medical Care", American Economic Review, 1963

9. Phelps CE, Parente ST. Priority setting in medical technology and medical practice assessment. Med Care. 1990; 28:703723.

10.Newhouse JP Controlled experimentation as research policy. In: Ginzberg E ed.) Health Services Research: Key to Health Policy. Harvard Univ. Press, 1991.

11.Keeler EB Journal of Medical Practice Management, v. 8, Summer 1992, pp. 317-321.

12.Williams A. The cost-benefit approach. Br. Med. Bull. 1974 30(3):252-56

13.Neumann PJ Goldie SJ, Weinstein MCPreferente-based measures in economic evaluation in healthcare Annu. Rev. Public Health. 2000. 21:587-611

14.Cutler DM, McLellan M Is Technological Change In Medicine Worth It? Health Aff 2001; 20(5); 11-29

15. Fisher ES, Wennberg DE, Stukel TA, Gottlieb DJ, Lucas FL, Pinder EL. The Implications of Regional Variations in Medicare Spending. Part 2: Health Outcomes and Satisfactions with Care. Ann Intern Med 2003 Feb 18; 138(4): 288-98. 
16. Informe sobre la Salud en el Mundo 2000. Mejorar el desempeño de los sistemas de salud. Organización Mundial de la Salud. Ginebra, 2000

17. Fetter RB, Youngsoo S, Freeman JL, Averill RF, Thompson JD. Case mix definition by Diagnosis-related Groups. Medical Care, 1980; 18: S1-S39

18. Fowles JB, Weiner JP, Knutson D, Fowler E Talking health status into account when setting capitation rates. A comparison of risk-adjustment methods JAMA, 1996; 276(16): 1316-21

19. Milgrom P, Roberts J. Economía, Organización y Gestión. Barcelona: Ariel, 1993.

20. Drummond MF, Stoddart GL, Torrance GW. Methods for the economic evaluation of health care programmes. Oxford, England: Oxford University Press; 1987
21. Kassirer J. Incorporating patients' preferences into medical decisions. New Engl J Med 1994; 330: 1895-1896.

22. Ortún V. Dimensión HUMANA 1997; 1(4): 17-23

23. Improving Medical Education: Enhancing the Behavioral and Social Science Content of Medical School Curricula Patricia A. Cuff, Neal Vanselow, Editors, Committee on Behavioral and Social Sciences in Medical School Curricula (Free Executive Summary) http://www.nap.edu/catalog/10956.html

24. World Federation for Medical Education (WFME) http://www.ifmsa.org/scome/wiki/index.php?title=World_Fede ration_for_Medical_Education_(WFME)

25. Declaración de Granada sobre estándares en la Educación Médica de Pregrado http://www.ugr.es/ facmed/tablon/granada.htm 